\title{
Inertia mobile mechanism research based on ADAMS
}

\section{Jiajia Chen ${ }^{\mathrm{a}}$, Qingming Wang ${ }^{\mathrm{b}}$, Jiachen $\mathrm{Ju}^{\mathrm{c}}$}

School of mechanical and power engineering, East China University of Science and Technology, Shanghai, China

a 18317052880@163.com , b wangqm@ecust.edu.cn, c jjc051126@live.cn

Keywords: Inertial force, dynamic characteristics, motion control

\begin{abstract}
In this paper, a new design of a mechanical device based on inertial force is presented. The theoretical analysis and some kinetics equations are established. The dynamic characteristics of mechanical device are greatly influenced by the coefficient of friction during the moving process. Relatively low coefficient of friction is good for the movement. A simulation model based on ADAMS to analyze the dynamic characteristics of a new mechanism driven by inertial force is built. The result of simulation is consistent with theoretical analysis, which proves the feasibility of mechanical device's motion and the correctness of the theoretical analysis.
\end{abstract}

\section{Introduction}

Traditional drive mode has complex structure and a relatively high cost [1]. Using the inertial force as drive mode, the new mechanical device can realize the low cost and compact structure. Using the inertia force to drive, the use of the transmission mechanism is reduced [2]. In this paper, the virtual prototype model is built before setting up experimental prototype, which providing guidance for manufacturing the experimental prototype.

\section{Dynamics analysis of robot}

\subsection{Introduce of structure and motion principle.}

New mechanical device is shown in figure 1, the main components are supporting blocks, scroll wheel, two small motors, connecting rod, mass, as well as the body and so on. Small motors drive the mass through the connecting rod, so centrifugal force is produced.

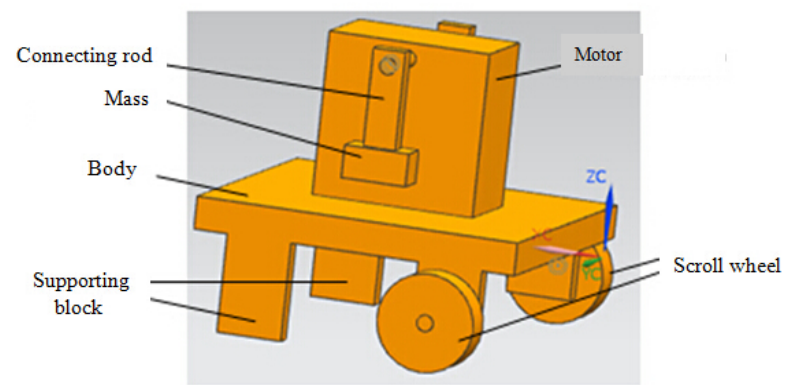

Figure 1 new mechanical device

When the block is on the side of scroll wheel, the centrifugal force will pull the body forward. At the same time, the centrifugal force can produce a clockwise torque. It is the torque that decreases the support force on the block, so the friction force decreases at the same time. Because the rotating motor is closer to the scroll wheel, the forward friction resistance is less than the backward friction resistance.

\section{2 analysis of dynamic model.}

The simplified model of Mobile mechanism is shown in figure 2. The body is simplified to a single block $\mathrm{M}$. The connecting rod length is represented by $\mathrm{r}$, regardless of its own weight. The mass block $m$ keep uniform rotation, speed value is $\omega$. The distance between the center of rotation and the bottom of body is represented by $\mathrm{H}$, and the distance between rotation center of connecting rod and the wheel is represented by L1, L2. 


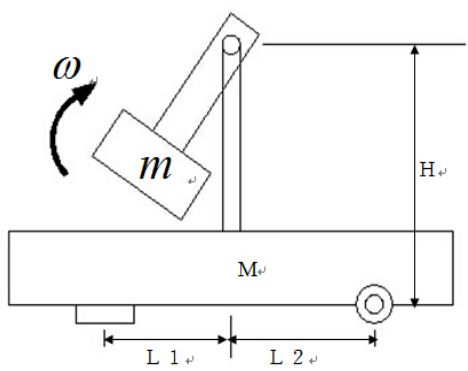

Figure 2 Simplified model

First of all, we only consider the kinetics equations of linear motion. Force analysis was carried out on the mass $\mathrm{m}$ and block $\mathrm{M}$ [3]. The force analysis diagram is shown in figure 3 . The kinetics equations of linear motion:

$$
\begin{aligned}
& \left\{\begin{array}{l}
F_{I} \cos \theta+F_{A x}=m \ddot{x} \\
F_{I} \sin \theta+m g-F_{A y}=0 \\
M_{A y}+m g r \cos \theta=0
\end{array}\right. \\
& \left\{\begin{array}{l}
-F_{A x}-f_{1}-f_{2}=M \ddot{x} \\
N_{1}+N_{2}-F_{A y}+M g=0 \\
N_{1}\left(L_{1}+L_{2}\right)-M_{A f}-F_{A x} H-F_{A y} L_{2}-M g L_{2}=0
\end{array}\right.
\end{aligned}
$$

Because the mass keep uniform rotation, according to Alembert principle, the centrifugal force can be seen as an inertial force $F_{I}$. Point $A$ on rotating rod can be considered as a hinge constraint with friction, providing constraint force $\boldsymbol{F}_{A x}$ and $\boldsymbol{F}_{A y}$, and the friction torque $\boldsymbol{M}_{A f}$. Body $\mathrm{M}$ gets supporting force $N_{1}$ from block support, supporting force $N_{2}$ from the rolling wheel, and friction $f_{1}, f_{2}$.
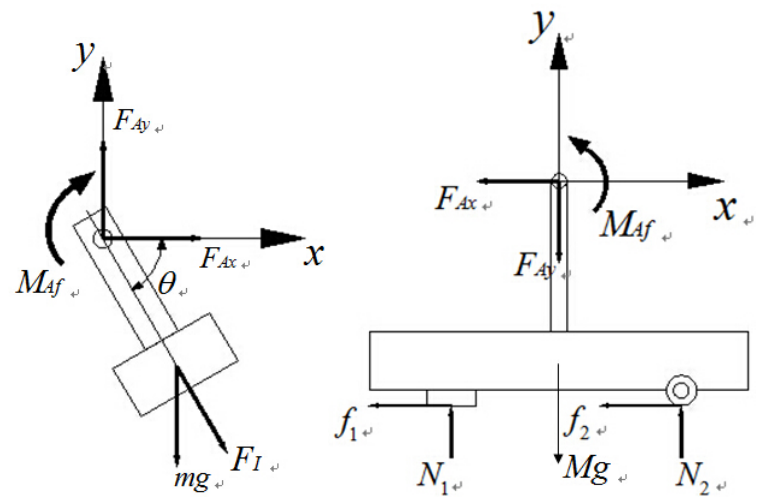

Figure 3 Force analyses

The rotation speed is set as $2 \mathrm{r} / \mathrm{s}$. We set the sliding friction between supporting block and ground as 0.1 . We can get the move trend with the kinetics equations with MATLAB. The mechanism's theoretical displacement-time curve is shown in figure 4.

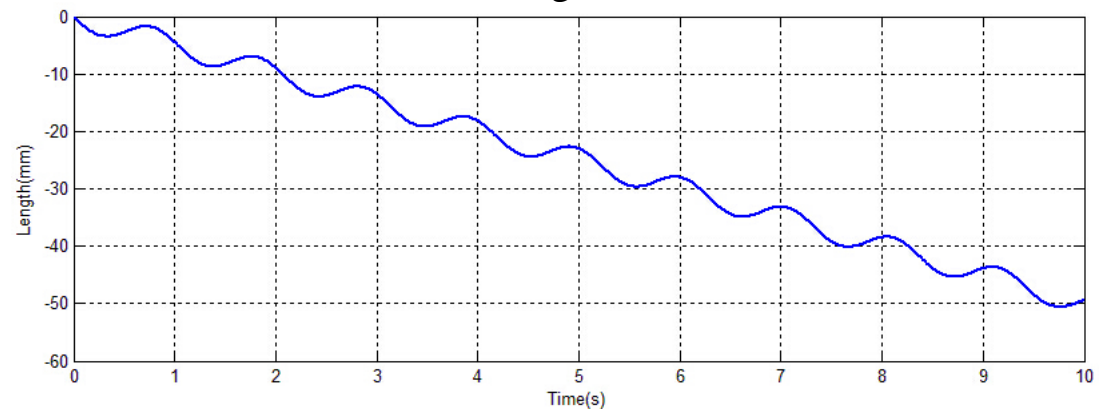

Figure 4 theoretical displacement-time curve 


\section{Simulation and analysis with ADAMS}

The dynamics analysis on new type inertial mobile mechanism shows the main parameters which impact the mechanism's movement characteristics, including rotation speed of motor, the weight of the block, the installation height of motor, and the distance between the scroll wheels and motor.

\subsection{Set up a virtual prototype.}

Using the graphical interface module of ADAMS, we can import the geometric model from UG into ADAMS [4], which will save the time to build a model. Firstly opening ADAMS/VIEW, we build ground part. There are a total of five moving parts. There are the body (fixed with two supporting blocks), two scroll wheel, two connecting rods (fixed mass).

\subsection{The results of simulation and analysis.}

To close the real situation, the coefficient of friction is set at every rotation constraint. We consider the rolling friction between ground and wheels, and the sliding friction between supporting block and ground. And the acceleration of gravity parameter is 9.8. To study the dynamic characteristics of mechanism, we measure the movement of mass center.

We start the simulation in the condition that the body keeps linear motion, and the two motors keep synchronous rotation. The rotation speed is set as $2 \mathrm{r} / \mathrm{s}$. The first time we set the sliding friction between supporting block and ground as 0.1.and the second time sliding friction is 0.5. The mechanism's level displacement - time curves are shown in figure 5.

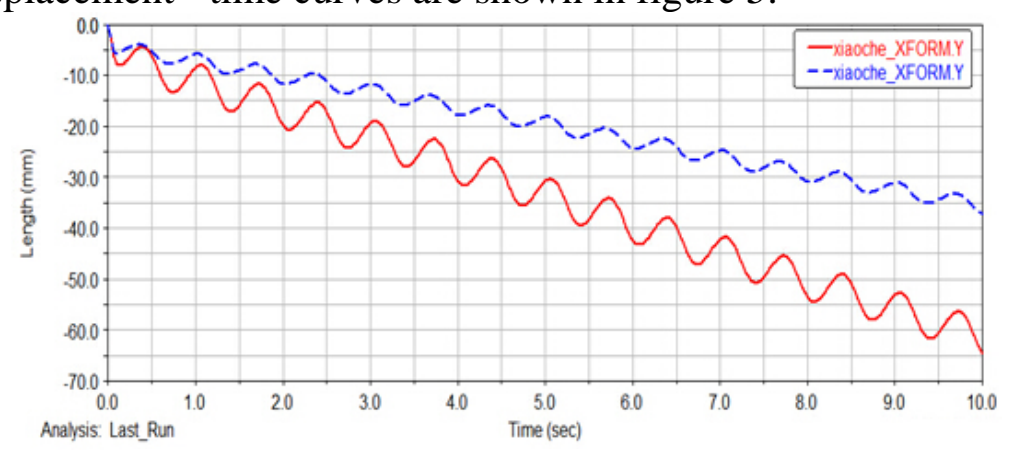

Figure 5 level displacement - time curves

We can find that the mechanism has both forward and backward movement, which is caused by the cycle change of centrifugal force. Because the trend of the forward is larger, the mechanism realizes forward movement in a whole cycle. The bigger the frictional coefficient is, the smaller the mechanism's advance distance is. Due to the particularity of centrifugal force, the mobile mechanism will still appear backward motion in the process of motion.

\section{Summary}

A new mechanical device driven by inertial force is analyzed in this paper. A simulation model based on ADAMS is built. We take simulation analysis under different coefficient of friction and find that the smaller friction coefficient is in favor of the movement of the body. The result of simulation is consistent with theoretical analysis, which proves the feasibility of mechanical device's motion and the correctness of the theoretical analysis. Virtual prototype based ADAMS is presented in this paper, providing the basis and the reference for the design of the test model.

\section{Acknowledgements}

This research was supported by National Natural Science Foundation of China (No.51175176)

\section{References}

[1] A Mobile Micro-Robot using Centrifugal Forces. Proceedings of the 1999 IEWASME, International Conference on Advanced Intelligent Mechatronics, September 19-23, 1999, Atlanta, 
USA

[2] HuaShunMing, guang-ming cheng, jun-wu kan, etc. The research progress of displacement of piezoelectric precision drive [J]. Journal of piezoelectric and acousto-optic, 2004, 26 (3) : 192-195. [3] zhang hui. University physics mechanics [M]. Beijing: Qinghua university press, 1999.

[4] Tiago Martinuzzi Bufiol, Sergio Seheer CAD and CAE integration through scientific visualization teehnlques for tiny illumination design [J]. Journal of Tsinghua Science and Technology. 2008.13 (1) Supp: 26-33. 